\title{
Unilateral Microphthalmos
}

National Cancer Institute

\section{Source}

National Cancer Institute. Unilateral Microphthalmos. NCI Thesaurus. Code C101190.

A congenital abnormality characterized by the presence of one abnormally small eye globe and one normally sized eye globe. 